\title{
REGRESSÃO ESPONTÂNEA DO CORPO LÚTEO EM BOVINOS - REVISÃO
}

\author{
SPONTANEOUS CORPUS LUTEUM REGRESSION IN CATTLE: REVIEW
}

\author{
G. PUGLIESI ${ }^{1 *}$, T. K. NISHIMURA ${ }^{2}$, G. D. MELO ${ }^{3}$, C. M. B. MEMBRIVE ${ }^{4}$, J. R. NAVES ${ }^{5}$, \\ G. R. CARVALHO
}

\begin{abstract}
RESUMO
O conhecimento do processo de luteólise dentro da fisiologia reprodutiva se torna importante para que ocorra a compreensão do reconhecimento materno da gestação e seja possível formular estratégias anti-luteolíticas que visem manter esta fase conferindo aumentos na eficiência reprodutiva e produtiva. Durante o processo de luteólise, o tecido luteal sofre mudanças bruscas na capacidade esteroidogênica, vascularização e remodelamento, resultando em substituição da glândula por tecido conjuntivo, sendo a PGF2 $\alpha$ o principal determinante na indução espontânea da luteólise em bovinos. Simultaneamente, ocorre um íntimo processo entre as relações anatômicas que compreende o ovário, útero e suas vascularizações. A exposição do útero à interação coordenada entre P4, E2 e ocitocina aliada à expressão de seus receptores são essenciais para a secreção pulsátil de PGF2 $\alpha$ pelo endométrio a qual induzirá a luteólise. Nesta regressão do corpo lúteo (CL), estão envolvidos ainda os hormônios (LH, prolactina, cortisol) e várias substâncias vasoativas (óxido nítrico, VEGF, EG-VEGF, bFGF, END-1, AngII). A concentração de PGF2 $\alpha$ pode ser representada pela mensuração sanguínea da concentração de seu metabólito 13,14-dihidro-15-ceto- PGF2 $\alpha$ (PGFM) sendo a pulsatilidade da secreção de PGF2 $\alpha$ durante os períodos pré-luteolítico, luteolítico e pós-luteolítico mensurável pelo aumento na concentração de PGFM. Neste artigo de revisão, será possível compreender e discutir os principais aspectos endócrinos e moleculares que ocorrem com as mudanças morfofuncionais no CL durante o processo de luteólise fisiológica em fêmeas bovinas.
\end{abstract}

PALAVRAS-CHAVE: Luteólise Espontânea, Bovinos.

\section{SUMMARY}

The knowledge of the luteolysis process within reproductive physiology becomes important to occur the understanding of maternal recognition of gestation being possible to formulate anti-luteolytic strategies aimed at maintaining this phase conferring increases in reproductive and productive efficiency. During the luteolysis process, luteal tissue undergoes abrupt changes in steroidogenic capacity, vascularization and remodeling, resulting in replacement of the gland by connective tissue, and PGF2 $\alpha$ is the main determinant in the spontaneous induction of luteolysis in cattle. Simultaneously, an intimate process occurs between the anatomical relationships that comprise the ovary, uterus and its vascularizations. The exposure of the uterus to the coordinated interaction between P4, E2 and oxytocin, together with the expression of its receptors, are essential for the pulsating secretion of PGF2 $\alpha$ by the endometrium, which will induce luteolysis. In this regression of the corpus luteum (CL), hormones (LH, prolactin, cortisol) and various vasoactive substances (nitric oxide, VEGF, EG-VEGF, bFGF, END-1, AngII) are also involved. The concentration of PGF2 $\alpha$ can be represented by the blood measurement of the concentration of its metabolite 13,14-dihydro-15-keto-PGF2 $\alpha$ (PGFM), being the pulsatility of PGF2 $\alpha$ secretion during the pre-luteolytic, luteolytic and post-luteolytic measured by the increased concentration of PGFM. In this review article, it will be possible to understand and discuss the main endocrine and molecular aspects that occur with the morphofunctional changes in CL during the physiological luteolysis process in bovine females.

KEY-WORDS: Luteolysis Spontaneous, Bovine.

\footnotetext{
${ }^{1}$ Departamento de Reprodução Animal, Faculdade de Medicina Veterinária e Zootecnia, Universidade de São Paulo, Pirassununga/SP, Brasil

${ }^{2}$ Departamento de Zootecnia, Faculdade de Zootecnia e Engenharia de Alimentos, Universidade de São Paulo, Pirassununga/SP, Brasil

${ }^{3}$ Departamento de Reprodução Animal, Faculdade de Medicina Veterinária e Zootecnia, Universidade de São Paulo, Pirassununga/SP, Brasil

${ }^{4}$ Universidade Estadual Paulista Júlio de Mesquita Filho (UNESP), Campus de Dracena/SP

${ }^{5}$ Departamento de Reprodução Animal, Faculdade de Medicina Veterinária e Zootecnia, Universidade de São Paulo, Pirassununga/SP, Brasil

${ }^{6}$ Departamento de Zootecnia, , Universidade Federal de Viçosa, Viçosa/MG, Brasil

* Este manuscrito foi redigido com texto extraído da tese de doutorado do primeiro autor. Pugliesi, G. Secreção de prostaglandinas antes, durante e após a luteólise em bovinos, 2012. 178 f. Tese (Doutorado em Zootecnia), Universidade Federal de Viçosa, Viçosa, 2012.
} 


\section{INTRODUÇÃO}

A fertilidade de um rebanho bovino afeta a produtividade e lucratividade da atividade pecuária, assim como a eficiência reprodutiva dos mesmos. Logo, para que se tenha o aumento nos índices de eficiência reprodutiva de um rebanho, é necessário compreender os mecanismos endócrinos, celulares e moleculares envolvidos nos eventos do ciclo estral,além dos manejos em que esses animais são expostos, pois o ambiente influencia diretamente nesses índices. Outro fator capaz de influenciar na fertilidade dos bovinos é a mortalidade embrionária, sendo uma das causas mais relevantes da baixa fertilidade, promovendo altas perdas econômicas na atividade pecuária (Diskin et al, 2006). As principais perdas embrionárias ocorrem no "período crítico" entre o $15^{\circ}$ e o $19^{\circ}$ dia após a ovulação (Diskin et al, 2006). Neste período, ocorre o reconhecimento materno da gestação que compreende mecanismos que visam à inibição da regressão do corpo lúteo (CL) (Bertan et al, 2006). O reconhecimento materno da gestação requer um ambiente uterino otimizado, o qual depende da função luteal em secretar adequadas quantidades de progesterona $(\mathrm{P} 4)$.

A lise ou regressão do $\mathrm{CL}$ (luteólise) determina a redução da secreção de $\mathrm{P} 4$ (fim da fase luteal) e é importante para que a fêmea bovina retorne a um novo ciclo reprodutivo após a não constatação de uma gestação pelo animal. O processo de luteólise envolve diversos mecanismos anatômicos, endócrinos e moleculares que estimulam a secreção de agentes luteolíticos, determinando o fim da funcionalidade e da estrutura física que compreendia o CL (Ginther et al,2012). Assim, o conhecimento do processo de luteólise dentro da fisiologia reprodutiva na vaca é importante não só para o controle farmacológico do ciclo estral, mas também para compreensão do reconhecimento materno da gestação e formulação de estratégias anti-luteolíticas que visem manter a gestação. Neste sentido, estudos de cunho fisiológico enfocando a elucidação dos mecanismos que governam a luteólise sendo conduzidos por indução, atraso ou bloqueio da luteólise (Pugliesi et al, 2011; 2012a; 2012b; Shrestha et al, 2011; Ginther et al, 2010) se constituem a base para o desenvolvimento de novas biotécnicas reprodutivas que incrementem o manejo reprodutivo e a fertilidade em bovinos.

Desta forma, objetiva-se com este artigo de revisão, compreender e discutir os principais aspectos endócrinos e moleculares nas mudanças morfofuncionais no CL durante o processo de luteólise fisiológica em fêmeas bovinas.

\section{CORPO LÚTEO E ESTEROIDOGÊNESE LUTEAL}

O CL é um órgão endócrino temporário cuja principal função é a secreção de $\mathrm{P} 4$ pelas células esteroidogênicas (Niswender et al, 2000; Skarzynski et al, 2008; Salles; Araujo, 2010). A cada ciclo estral, as células luteínicas esteroidogênicas sintetizam e liberam
P4 na circulação sistêmica, promovendo a quiescência na contratilidade do miométrio, o desenvolvimento glandular do endométrio e o ambiente uterino adequado para o desenvolvimento do concepto. A adequada função luteal em secretar P4 é crucial para determinar a duração fisiológica do ciclo estral e para ocorrer uma gestação bem sucedida.

O tecido luteal é composto por vários tipos celulares heterogêneos que consistem não apenas de células esteroidogênicas (células luteais pequenas e grandes), mas também de células não esteroidogênicas (células endoteliais, células musculares, periócitos, fibrócitos e células imunes (Shirasuna et al, 2007)). As células endoteliais vasculares representam mais de $50 \%$ do total do número de células no CL (O'Shea et al, 1989; Lei et al, 1991) e são responsáveis pela secreção de muitas substâncias vasoativas que regulam diretamente a secreção de P4 luteal (Myamoto et al, 1993, 1997; Shirasuna et al, 2007). Assim, os vasos sanguíneos e as células endoteliais no CL têm um papel essencial na função luteal nas vacas. O CL bovino se desenvolve rapidamente entre 2 a 3 dias após a ovulação, acompanhado de grande angiogênese e vascularização (Miyamoto et al, 2009). A maioria das células esteroidogênicas está em íntimo contato com os capilares. Fatores de crescimento tais como: fator de crescimento endotelial vascular, do inglês vascular endotelial growth factor (VEGF) e o fator de crescimento fibroblástico básico, do inglês basic fibroblast growth factor (bFGF), são os principais atuantes no desenvolvimento e na manutenção da densa rede de capilares neoformados e contribuem para a produção de P4 (Sales ; Araujo, 2010).

A esteroidogênese luteal é estimulada principalmente pelo LH (Skarzynski et al, 2008), hormônio necessário para o desenvolvimento estrutural e funcional do CL. O estímulo do crescimento e da função luteal, também realizado por hormônios como a ocitocina, prostaglandinas e fatores de crescimento (Niswender et al, 2000). Após a ovulação, para que haja secreção de P4 em grandes quantidades pelas células luteais, é necessário que haja diferenciação celular, aumento na expressão de enzimas para a conversão do colesterol em $\mathrm{P}_{4}$ e das proteínas transportadoras de colesterol para o interior da membrana da mitocôndria. O colesterol transportado para este local interage com a enzima P450scc, que o transforma em pregnenolona (Bertan et al, 2006). O retículo endoplasmático liso transporta este precursor e faz a conversão em P4 por ação da enzima ßhidroxiesteróide deidrogenase ( $\Delta 5 \Delta 4$ isomerase-3ßHSD) A secreção de $\mathrm{P}_{4}$ pelo $\mathrm{CL}$ é caracterizada por aumento na expressão das enzimas envolvidas na conversão de colesterol em $\mathrm{P}_{4}$ (P450scc e 3ß-HSD) e por decréscimo na nas enzimas que convertem $\mathrm{P}_{4}$ em $\mathrm{E}_{2}$ (P45017 $\alpha$-hidroxilase e $\mathrm{P} 450$ aromatase) (Bertan et al, 2006).

\section{LUTEÓLISE ESPONTÂNEA EM BOVINOS}

Na ausência da fertilização ou na incapacidade do concepto em sinalizar sua existência no útero, o CL 
deve regredir para possibilitar a ocorrência de um novo estro (Milvae, 2000; Webb et al, 2002). Durante o processo de lise ou regressão do CL (luteólise) o tecido luteal sofre mudanças bruscas na capacidade esteroidogênica, vascularização e remodelamento, resultando em substituição da glândula por tecido conjuntivo (Stocco et al, 2007). Assim, a luteólise espontânea pode ser dividida em duas fases (Stocco et al, 2007; Skarzynski et al, 2008): a luteólise funcional, a qual é associada a um marcado decréscimo na secreção de P4,e a luteólise estrutural, que ocorre após o início do declínio da P4 e é determinada pela apoptose celular do CL até formação do corpo albicans.

\section{LUTEÓLISE FUNCIONAL}

A luteólise funcional é caracterizada pela redução da secreção de $\mathrm{P}_{4}$ em um curto período de tempo na ovelha (McCracken et al, 1999) e na vaca (Ginther et al, 2010a). A luteólise funcional se inicia antes de se observar mudanças morfológicas na integridade das células luteais (Stocco et al, 2007). Assim, considerando-se as concentrações plasmáticas de P4, pode-se classificar três períodos relacionados com a luteólise espontânea em bovinos (Ginther et al,2010a; 2010b), sendo eles, a) o período antes do início da redução da secreção de P4 (pré-luteólise), b) o período de grande redução da P4 (período luteolítico) e c) o período após o decréscimo das concentrações de P4 a $<1 \mathrm{ng} / \mathrm{mL}$ (período pós-luteolítico). Baseado em amostragem da $\mathrm{P}_{4}$ plasmática de hora em hora, estimase que a duração do período luteolítico é de 24 horas em novilhas (Ginther et al, 2010a).

A redução das concentrações de $\mathrm{P} 4$ durante a luteólise funcional em bovinos não ocorre de maneira contínua como observado na espécie equina (Ginther et al, 2011a; 2011b). Em novilhas, aumentos ou flutuações nas concentrações plasmáticas de P4 ocorrem ao longo do período luteolítico (Ginther et al, 2010a; 2011b; 2011c). Há evidências em ratos (revisado em Stocco et al, 2007) indicando que durante a regressão funcional, as células luteais estão ainda com atividade esteroidogênica, entretanto o principal esteróide secretado durante este período não se constitui a $\mathrm{P}_{4}$, mas sim um metabólito da $\mathrm{P}_{4}(20 \alpha-$ progesterona). Em bovinos, outra característica que também pode ser observada durante a luteólise funcional é a perda de receptores de $\mathrm{LH}$ aliado a uma menor perfusão sanguínea no CL (Niswender et al, 1976). Esta redução na perfusão sanguínea luteal parece ocorrer após um aumento agudo e transitório no fluxo sanguíneo logo após a indução da luteólise com PGF2 $\alpha$ exógena (Acosta et al, 2002). Da mesma forma, durante um pulso luteolítico espontâneo de PGFM, a perfusão sanguínea luteal aumenta gradativamente durante a parte ascendente do pulso até o seu pico, e mantém elevado por 2 horas durante a parte descendente do pulso, para posteriormente reduzir (Ginther, 2007). Da mesma forma, durante pulsos de PGFM simulados por infusão intrauterina com PGF2 $\alpha$, a perfusão sanguínea no CL aumenta dentro de 1 hora após o início da infusão em novilhas (Shrestha et al, 2010b; 2011).

Devido aos vários efeitos adversos e respostas não fisiológicas após a administração de doses farmacológicas de $\mathrm{PGF}_{2 \alpha}$ para induzir a luteólise (Ginther et al, 2009a; 2009b), o estudo das características da luteólise espontânea em bovinos deve ser realizado utilizando-se doses fisiológicas de $\mathrm{PGF}_{2 \alpha}$ e seus análogos, e em tempo e intervalos que se assemelham aos pulsos espontâneos. Um exemplo destes efeitos é que a administração exógena de $\mathrm{PGF}_{2 \alpha}$ promove um início mais rápido e reduz a duração da luteólise funcional em ovinos (McCracken et al, 1984) e bovinos (Ginther et al, 2009a). A administração de doses fisiológicas de $\mathrm{PGF}_{2 \alpha}$ em intervalos próximos aos intervalos dos pulsos espontâneos de $\mathrm{PGF}_{2 \alpha}$ induziu a luteólise funcional completa $(\mathrm{P} 4<1 \mathrm{ng} / \mathrm{mL})$ em ovelhas (Schramm et al, 1983) e novilhas (Ginther et al, 2009a). A dose total de $\mathrm{PGF}_{2 \alpha}$ requerida para causar a luteólise funcional quando administrada de maneira pulsátil é de $1 / 10$ a $1 / 40$ da dose mínima requerida quando administrado de maneira contínua (Schramm et al, 1983; Ginther, 2007). Aboelenain et al(2015) sugerem que o processo de regressão luteal envolve o processo de apoptose e alguns outros fatores como um aumento na expressão de atividade lisossomal, síntese de catepsina lisossomais e formação de fagolisossomos, indicando uma comunicação entre a função lisossomal, autofagia e apoptose durante a regressão do CL bovino.

\section{LUTEÓLISE ESTRUTURAL}

A regressão estrutural do CL é caracterizada pelo decréscimo no tamanho e peso da glândula, a qual eventualmente se torna um tecido totalmente regredido dentro do estroma ovariano denominado corpo albicans. A involução do CL é na verdade não somente a apoptose do tecido luteal, mas também a reposição do suporte vascular e tecido conectivo com feixes de fibras colágenas, fibroblastos e macrófagos. A maioria dos corpos albicans é substituída por estroma ovariano (Stocco et al, 2007). Na maior parte das espécies, a distinção entre a luteólise functional e estrutural não está claramente reportada e os dois eventos podem não ser inteiramente separados (McCracken et al, 1999). Por exemplo, em ratos a $\mathrm{PGF}_{2 \alpha}$ não causa a luteólise estrutural, a qual é induzida pela prolactina apenas após finalizada a luteólise funcional (Behrman et al, 1993). Em bovinos, a luteólise estrutural se inicia logo após o início do declínio da $\mathrm{P}_{4}$ plasmática (Ginther, 2007). Entretanto, o processo final de apoptose e substituição do tecido luteal pode demorar ainda alguns dias para ser completado após o fim da luteólise funcional (McCraken et al, 1999).

Uma grande variedade de agentes tem sido proposta como mediadores da luteólise estrutural (McCracken et al, 1999). Macrófagos e outras células imunes possuem papel importante na regressão luteal via a liberação do fator de necrose tumoral- $\alpha$ (TNF- $\alpha$ ) e outras citocinas (Benyo; Pate, 1992). O TNF- $\alpha$ pode também estar envolvido no efeito antiesteroidogênico durante a luteólise, já que in vitro o TNF- $\alpha$ inibe a 
produção de $\mathrm{P}_{4}$ estimulada pelo $\mathrm{LH}$ nas células luteais bovinas (Benyo; Pate, 1992).

Durante a luteólise estrutural, um remodelamento considerável do tecido luteal ocorre durante a transformação do CL para corpo albicans. As metaloproteinases têm sido sugeridas como as mediadoras do remodelamento tecidual, particularmente da matriz extracelular (BirkedalHansen, 1995). O CL produz inibidores específicos das metaloproteinases que auxiliam na manutenção da integridade estrutural da glândula. A mais abundante metaloproteinase no CL de bovinos (Smith et al, 1996) é a metaloproteinase-1 (TIMP-1). Na vaca, a expressão luteal da TIMP-1 aumenta em 24 horas após a indução da luteólise com $\mathrm{PGF}_{2 \alpha}$ (Smith et al, 1996). O efeito positivo da $\mathrm{PGF}_{2 \alpha}$ sobre a ação de algumas caspases e em citocinas coorelacionadas com a defragmentação de DNA celular também tem sido associado aos mecanismos que controlam a sinalização apoptótica durante a luteólise estrutural (revisado em Stocco et al, 2007).

\section{MECANISMOS ANATÔMICOS, ENDÓCRINOS E MOLECULARES ENVOLVIDOS NA LUTEÓLISE EM BOVINOS}

$\mathrm{O}$ entendimento dos mecanismos luteolíticos requer o conhecimento das relações anatômicas entre o ovário, útero e suas vascularizações associadas. A exposição do útero à interação coordenada entre P4, E2 e ocitocina (Hooper et al, 1986; Lefrance; Goff, 1988; Silvia et al, 1991) aliada à expressão de seus receptores (Mann et al, 2001; Mann; Lamming, 2006) são essenciais para a secreção pulsátil de PGF2 $\alpha$ pelo endométrio a qual induzirá a luteólise (McCracken, 1999). Além do E2, P4 e ocitocina, outros hormônios (LH, PRL, cortisol) e várias substâncias vasoativas (óxido nítrico, VEGF, EG-VEGF, bFGF, END-1, AngII) estão envolvidas no processo de regressão do CL (Stocco et al, 2007).

Neste contexto, verifica-se que o útero apresenta papel fudamental no controle da luteólise. A importância uterina foi primeiramente reportada em roedores (Loeb, 1923; 1927), através da inexistência de ciclos reprodutivos e a persistência do CL após a histerectomia. Efeitos similares também foram observados em ovinos, bovinos, suínos e equinos (Anderson et al, 1969). Já em primatas, este efeito não é observado (Beavis et al, 1969; Neill et al, 1969). A persistência luteal após a histerectomia nas espécies supracitadas indicou que o útero é o responsável pela produção de uma substância que causa a regressão cíclica do CL. Todas as espécies que mantiveram o CL após esse procedimento possuem um útero bicornuado (McCracken et al, 1999). Na maioria destes animais, o efeito unilateral da histerectomia foi observado, ou seja, manutenção do CL após retirada de apenas um corno uterino. Posteriormente, evidências indicaram que a PGF2 $\alpha$ é esta substância luteolítica, a qual é liberada ciclicamente pelo útero por volta dos dias 15 , 16 ou 17 após a ovulação (Thatcher et al, 2001), e é o principal determinante na indução espontânea da luteólise em bovinos (McCracken, 1999). A concentração de PGF2 $\alpha$ pode ser representada pela mensuração da concentração de seu metabólito 13,14dihidro-15-ceto- PGF2 $\alpha$, PGFM (Ginther,2007).

Um mecanismo local de transferência da PGF2 $\alpha$ existe no pedículo útero-ovariano da vaca (Ginther et al, 1966; 1967; Hixon; Hansen, 1974). Estudos envolvendo a ligação dos vasos sanguíneos do útero e do ovário indicaram a importância da via vascular no efeito local do útero sobre a função ovariana em diversas espécies (revisado em (McCracken et al, 1999)). A artéria ovariana é fortemente anexada à superfície da veia útero-ovariana e percorre a veia de forma tortuosa antes de sua entrada no hilo ovariano. Esta via local facilita que substâncias difundam da veia útero-ovariana para a artéria ovariana e alcançem o ovário diretamente sem passar pela circulação sistêmica (Ginther, 1981). Além desse mecanismo de ação local, há evidências de que a PGF $2 \alpha$ possa agir, em parte, pela via sistêmica na vaca, já que ciclos estrais foram observados em bovinos de corte após a hemi-histerectomia quando o lado que o CL estava presente não foi considerado (Ward et al, 1976). Adicionalmente, ciclos estrais de duração normal foram observados quando o ovário foi transplantado através de anastomoses vasculares com a artéria carótida e a veia jugular na vaca, e o outro ovário removido (Barcikowski et al, 1976).

Outra evidência que demonstra a maior eficácia da via local na indução da luteólise está relacionada à dose de PGF $2 \alpha$ necessária para induzir a regressão do CL. A dose mínima de PGF2 $\alpha$ (1-2 mg) quando administrada via intrauterina (IU) no corno uterino ipsilateral ao CL é em torno de 10 vezes menor do que a dose via sistêmica (15 mg), a qual é administrada via endovenosa (Louis et al, 1974; Lauderdale; Fokolowsky, 1979). Neste contexto, a injeção de PGF2 $\alpha(6 \mathrm{mg})$ dentro do corno uterino é seguido por maiores concentrações de PGF2 $\alpha$ na artéria ovariana ipsilateral do que na artéria carótida (Hixon ; Hansel, 1974). Assim, a rota natural de transporte entre o útero e ovário deve ser considerada em estudos da luteólise que utilizem PGF2 $\alpha$ exógena. Além disso, há a complexidade de se utilizar a veia jugular para tratamento com PGF2 $\alpha$ ou para amostragem sanguínea já que $65 \%$ da PGF2 $\alpha$ é metabolizada durante uma única passagem pelos pulmões na vaca (Davis et al, 1985).

\section{AÇÃO DA PROSTAGLANDINA F2 $\alpha$}

A secreção de PGF2 $\alpha$ pelo útero determina o final da fase luteal em várias espécies domésticas (McCracken et al, 1999; Weems et al, 2006; Skarzynski et al, 2008). Em bovinos, a secreção de PGF2 $\alpha$ ocorre em vários pulsos (4-7 pulsos) de curta duração (3-6 horas) durante 2 a 3 dias, ocorrendo previamente, durante e após o declínio da P4 circulante (Kindhal et al, 1981; Ginther, 2007; 2010b). Pulsos sequenciais de PGF2 $\alpha$ são requeridos para induzir a luteólise completa em bovinos (Ginther et al, 2009a), equinos (Ginther et al, 2009b) e ovinos (Schramm et al, 1983). A necessidade do estímulo sequencial da PGF2 $\alpha$ para promover a luteólise foi demonstrada em 
bovinos (Ginther et al, 2009a) através da ocorrência de luteólise completa $(\mathrm{P} 4<1 \mathrm{ng} / \mathrm{mL})$ após três sessões de infusão de PGF2 $\alpha(0,5 \mathrm{mg}$ via IU a cada 12 horas $)$, sendo que as concentrações de $\mathrm{P} 4$ resurgem quando realizado apenas uma sessão.

Devido aos vários efeitos adversos e respostas não fisiológicas após a administração de doses farmacológicas de PGF2 $\alpha$ para induzir a luteólise (Ginther et al, 2009a; 2009b), o estudo das características da luteólise espontânea em bovinos deve ser realizado utilizando-se doses fisiológicas de PGF $2 \alpha$ e seus análogos, e em tempo e intervalos que se assemelham aos pulsos espontâneos. Um exemplo desses efeitos é a administração de uma única dose luteolítica de PGF2 $\alpha$ que promove um início mais rápido com redução da duração normal da luteólise funcional em ovinos (McCracken et al, 1984) e bovinos (Ginther et al, 2009a).

A pulsatilidade da secreção de PGF2 $\alpha$ durante os períodos pré-luteolítico, luteolítico e pós-luteolítico pode ser mensurável pelo aumento na concentração de PGFM (Ginther, 2007). Durante o período luteolítico, os pulsos espontâneos de PGFM são mais proeminentes (pico e amplitude dos pulsos são maiores) do que os pulsos durante os períodos pré e pós-luteolítico (Ginther et al, 2010a; 2010b). Durante a parte ascendente do último pulso de PGFM no período pré-luteolítico, a concentração de $\mathrm{P} 4$ decresce para o mais baixo nível no pico do pulso de PGFM, seguido por um aumento de $\mathrm{P} 4$ (efeito rebote) para as concentrações prévias ao pulso (Ginther et al, 2010c; 2011c). O efeito rebote completo durante um pulso de PGFM no período pré-luteolítico é semelhante ao rebote com P4 que ocorre durante um pulso de PGFM induzido pelo E2 (Ginther et al, 2010d; Imam et al, 2010), e também é similar ao rebote completo que ocorre uma ou duas horas após o início da infusão de PGF2 $\alpha$ para simular o pulso de PGFM (Shrestha et al, 2010a; 2010b; 2011). Durante o início do período luteolítico, a P4 também decresce durante a parte ascendente do pulso de PGFM, entretanto, a concentração de P4 durante o rebote não retorna para as concentrações prévias (Ginther et al, 2010a; 2010c). No período luteolítico avançado o efeito rebote não é mais observado. O pico do último pulso de PGFM durante a pré-luteólise ocorre em média 4 horas (variação de 2 a 8 horas) antes da hora de transição entre pré-luteólise e luteólise e o primeiro pulso durante a luteólise ocorre 9 horas após a transição (Ginther et al, 2011c). Desta maneira, estes estudos indicam que o último pulso de PGF2 $\alpha$ durante a préluteólise reduz temporariamente a secreção de P4 e inicia o subsequente início da luteólise.

O sinal luteolítico promovido pela secreção inicial de PGF2 $\alpha$ promove uma cascata luteolítica envolvendo uma série de reguladores da função luteal, como o fluxo sanguíneo local, citocinas e fatores de crescimento. $\mathrm{O}$ efeito inicial da PGF2 $\alpha$ na esteroidogênese luteal é mediado pelo receptor de membrana ligado a proteína $\mathrm{G}$ (Davis; Rueda, 2002). A ativação desse receptor pela PGF2 $\alpha$ promove a produção de inusitol trifosfato (IP3) e diaciglicerol (DAG) a partir do fosfatidilinositol-4,5-difosfato mediado pela ação da fosfolipase C (Davis et al, 1987). O aumento do IP3 e do DAG é seguido pelo aumento do cálcio intracelular livre e atividade da proteína quinase C (Davis et al, 1987). Adicionalmente à ativação da fosfolipase $\mathrm{C}$, há evidências de que a PGF2 $\alpha$ ativa a via da fosfolipase D, produzindo ácido fosfatídico em adição ao DAG, e ativa a cascata sinalizadora da MAP quinase (Tai et al, 2001; Chen et al, 2001).

O decréscimo rápido do fluxo sanguíneo luteal tem sido proposto como uma das principais ações da PGF2 $\alpha$. Mudanças rápidas nos fatores angiogênicos diretamente envolvidos com o início da regressão vascular e modulação da secreção de P4 são causadas pela PGF2 $\alpha$ (Miyamoto et al, 2009). Inicialmente, o fluxo sanguíneo luteal na área periférica do CL aparentemente é aumentado rapidamente como resultado da vasodilatação causada pelo óxido nítrico (Acosta et al, 2002; Miyamoto et al, 2009). Isto sugere que o aumento do fluxo sanguíneo luteal causado pela PGF2 $\alpha$ é um dos primeiros sinais do início da cascata luteolítica na vaca. $\mathrm{Na}$ fase inicial da luteólise, a PGF2 $\alpha$ suprimi drasticamente a expressão de VEGF no CL. Outras substâncias vasoativas, como a EDN1, Ang II e a PGF $2 \alpha$ de origem luteal aumentam no CL, aonde uma severa vasoconstricção é induzida para interromper o suporte sanguíneo e acelerar a cascata luteolítica (revisado em (Miyamoto et al, 2009)).

O mecanismo pelo qual a PGF $2 \alpha$ reduz os níveis de P4 secretado pelo CL ainda precisa ser mais bem elucidado em bovinos. Em ratos, tem sido demonstrado (Strauss; Stambaugh, 1974; Kawano et al, 1988) que a PGF2 $\alpha$ não inibe a síntese de $\mathrm{P} 4$, mas ao invés, causa o seu metabolismo para $20 \alpha$-di-hidroprogesterona. A administração de PGF $2 \alpha$ exógena em bovinos causa a redução de RNAm para fatores relacionados a síntese esteroidogênica (StAR, 3 $\beta$ hidroxiesteróide dehidrogenase - $3 \beta$-HSD) e fatores agiogênicos (VEGF, Ang1, IGF-I e IGF-II) e aumenta o RNAm para fatores relacionados a síntese de PGs (COX-2 e enzima prostaglandina sintase (Shirasuna, 2010)). O efeito positivo da PGF2 $\alpha$ sobre a ação de algumas caspases e em citocinas correlacionadas com a desfragmentação de DNA celular tem sido associado aos mecanismos que controlam a sinalização apoptótica durante a luteólise estrutural (revisado em (Stocco et al, 2007)). Desta maneira, a ação da PGF2 $\alpha$ na luteólise é mediada por fatores locais que atuam na síntese de P4 e na regressão estrutural das células luteais (Skarkzynski et al, 2008).

\section{PAPEL DOS HORMÔNIOS ESTEROIDAIS E DA OCITOCINA}

O E2, P4 e a ocitocina têm sido correlacionados como reguladores do início da secreção de PGF2 $\alpha$ durante a luteólise em fêmeas bovinas. Atualmente, vários modelos de mecanismos controladores da luteólise têm sugerido mudanças na expressão endometrial de receptores de E2, P4 e ocitocina influenciando na regulação da secreção de PGF $2 \alpha$ uterina e consequente luteólise na vaca (Silvia et al, 1991; Arosh et al, 2004; Weems et al, 2006). 
Nestes modelos, a expressão de receptores de E2 e de ocitocina é suprimida na fase inicial e intermediária de desenvolvimento do CL devido ao efeito inibitório da alta concentração de P4 (Wathes; Hamon, 1993; Ivell et al, 2000). O efeito da P4 sobre a sensibilidade uterina à ocitocina envolve uma ação não genômica da P4 no receptor uterino de ocitocina. A P4 se liga ao receptor de ocitocina com alta afinidade, inabilitando a molécula de ocitocina ter capacidade de se ligar e, consequentemente, suprimindo a indução da produção de IP3 e mobilização de cálcio necessários para a resposta celular (Zingg et al, 1998). A exposição do útero à $\mathrm{P} 4$ por no mínimo 10 dias após a ovulação é necessário para ativar os mecanismos de secreção espontânea de PGF2 $\alpha$ (McCracken et al, 1984; Silvia; Raw, 1993). A prolongada exposição à P4 promove o acúmulo nas células endometriais de ácido araquidônico e COXs necessárias para síntese de PGF $2 \alpha$.

Durante o final da fase luteal, os receptores de E2 no útero aumentam após o decréscimo dos receptores de $\mathrm{P} 4$ e decréscimo da sensibilidade uterina à P4 (Meyer et al, 1988). Sequencialmente, a ação do E2 circulante sobre os seus receptores endometriais estimula a síntese de receptores de ocitocina com subsequente secreção de PGF2 $\alpha$ através da ação da ocitocina em seus receptores endometriais (Flint; Sheldrick, 1985; Silvia et al, 1991; Mann et al, 2001). A fonte de E2 durante a luteólise é oriundo dos folículos ovarianos, especialmente os de maior diâmetro e/ou futuros folículos dominantes que irão se desenvolver para folículos ovulatórios (Beg; Ginther, 2006). Na vaca, o aumento inicial de receptores de ocitocina que precede a luteólise ocorre após o $13^{\circ}$ dia pós-ovulação (Mann; Lammming, 1994).

$\mathrm{O}$ aumento da proeminência (pico e amplitude) dos pulsos de PGF2 $\alpha$ após a transição entre a preluteólise e luteólise (Ginther et al, 2010a; 2010b) é temporariamente associado com o aumento na concentração de E2 circulante (Ginther et al, 2010c; 2011c). A secreção de PGF2 $\alpha$, representada pelas concentrações de PGFM, é induzida dentro de 6 a 8 horas após o tratamento com E2 exógeno em novilhas (Thatcher et al, 1986; Araujo et al, 2009). Um único tratamento com $0.1 \mathrm{mg}$ de E2 no dia 14 pós-ovulação (Ginther et al, 2010d; Iman et al, 2010) estimula um pulso proeminente de PGFM e a luteólise prematura.

Em ruminantes, pulsos de ocitocina (ou da sua neurofisina associada) ocorrem concomitantes com pulsos de PGF2 $\alpha$ durante a luteólise (Fairclough et al, 1980; Webb et al, 1981). A maioria destes pulsos de ocitocina aparentemente é secretada pelo CL (Walters et al, 1984; Hooper et al, 1986; Moore et al, 1986). A PGF2 $\alpha$ secretada pelo útero e a ocitocina luteal compõem um sistema de retroalimentação positiva. Em bovinos, a administração de PGF2 $\alpha$ estimula a secreção de ocitocina no CL (Flint; Sheldrick, 1982; Lamsa et al, 1989), assim como altas doses de ocitocina promovem o aumento da secreção de PGF2 $\alpha$ (Silvia; Taylor, 1989; Kotwica et al, 1998). Entretanto, os fatores que iniciam e terminam a secreção destes hormônios ainda não foram determinados.
Durante os pulsos espontâneos de PGF2 $\alpha$, a concentração de PGF2 $\alpha$ no efluente venoso úteroovariano aumenta antes de qualquer detectação do aumento das concentrações de ocitocina (Moore et al, 1986). Isto implica que apesar de não se saber o que promove o início da secreção de PGF2 $\alpha$, a ativação do sistema de retroalimentação positiva inicia-se no lado uterino. Duas possibilidades têm sido estudadas como causa do estímulo da secreção inicial de PGF2 $\alpha$. A primeira considera que o útero possui um centro rítmico endógeno que promove a secreção de PGF2 $\alpha$ a cada 6-8 horas de intervalo. A outra possibilidade é que o útero recebe um sinal pulsátil externo oriundo de outro tecido. $\mathrm{O}$ principal candidato em prover essa sinalização é a ocitocina secretada pela hipófise posterior (Silvia et al, 1991). Apesar da sincronização dos pulsos de PGF2 $\alpha$ e ocitocina, a precisa relação temporal da ocitocina secretada pela hipófise posterior e da PGF2 $\alpha$ uterina ainda não foi caracterizada.

Com base nestes aspectos relacionados acima, foi proposto por McCracken et al (1995) um modelo hipotético que explica como ocorreria o controle neuroendócrino da secreção pulsátil de PGF2 $\alpha$ durante a luteólise. Modelo: 1) No período luteal tardio, a perda da ação da $\mathrm{P} 4$ ocorre devido à redução da expressão de receptores de P4 tanto no hipotálamo quanto no endométrio, o que resulta no retorno da ação do E2 sobre estes tecidos; 2) O retorno da ação estrogênica irá estimular o centro hipotalâmico gerador dos pulsos de ocitocina a secretá-la em pulsos de alta frequência e baixa concentração e, simultaneamente, estimular a expressão de receptores de ocitocina no útero; 3) Baixos níveis de PGF2 $\alpha$ (subluteolítico) serão secretados pelo útero devido a interação da ocitocina de origem hipofisária com os seus receptores endometriais; 4) Baixas concentrações de PGF2 $\alpha$ são suficientes para iniciar a liberação de ocitocina luteal através da ação sobre os receptores de alta sensibilidade; 5) A secreção de ocitocina suplementar irá amplificar a secreção de PGF2 $\alpha$ endometrial; 6) A secreção de PGF $2 \alpha$ pelo endométrio será, neste momento, alta o suficiente para ativar os receptores de PGF2 $\alpha$ de baixa sensibilidade e irá inibir a secreção de P4 e liberar mais ocitocina do CL. Tais hipóteses ainda precisam ser melhores estudadas em fêmeas bovinas.

\section{AÇÃO DO HORMÔNIO LUTEINIZANTE}

O efeito negativo da secreção de PGF2 $\alpha$ sobre a concentração de P4 é balanceado contra um efeito positivo das oscilações de LH durante o período final da preluteólise e período inicial da luteólise (Ginther et al, 2010c; 2011c). O balanço periódico entre o efeito luteolítico da PGF2 $\alpha$ e o luteotrófico do LH é compatível com os seguintes achados em novilhas: 1) o LH exógeno prolonga a vida luteal (Donaldson; Hansel, 1965); 2) o LH é essencial para secreção de P4 pelo CL (Quintal-Franco et al, 1999); 3) aumento das concentrações de LH durante o rebote de $\mathrm{P} 4$ em pulsos de PGFM induzidos pelo E2 (Imam et al, 2010); e 4) o LH aumenta após a infusão de PGF2 $\alpha$ (Shrestha et al, 2010b; 2011). Durante a fase intermediária do desenvolvimento luteal, um pulso de LH com pico de 
baixa frequência e alta amplitude ocorre a cada 4 horas (Cupp et al, 1995), e é associado com um aumento na P4 (Procknor et al, 1986). A ritmicidade entre as oscilações de P4 e LH durante a preluteólise é perdida e a proeminência das flutuações de P4 são reduzidas quando as oscilações de LH são bloqueadas por um antagonista de GnRH (Ginther et al, 2011b).

$\mathrm{O}$ estímulo do LH na secreção de P4 não se torna eficiente no transcorrer da transição entre preluteólise e luteólise. Isto é indicado pela redução na ocorrência do pico de LH no mesmo momento que o pico da flutuação de P4 ocorre na luteólise (29\%) comparado com a preluteólise (77\%) e pela não observação do efeito rebote completo nas concentrações de P4 após o pico de PGFM e associado temporalmente ao aumento de LH no período luteolítico (Ginther et al, 2010e). A redução na sincronia entre os picos dos pulsos de LH e das flutuações de P4 durante a luteólise pode refletir a menor responsividade ao $\mathrm{LH}$ das células luteais em regressão.

A interação do LH em relação às concentrações do E2, ocitocina e PGF2 $\alpha$ e seu efeito na secreção de P4 estão ilustrados na Figura 1.

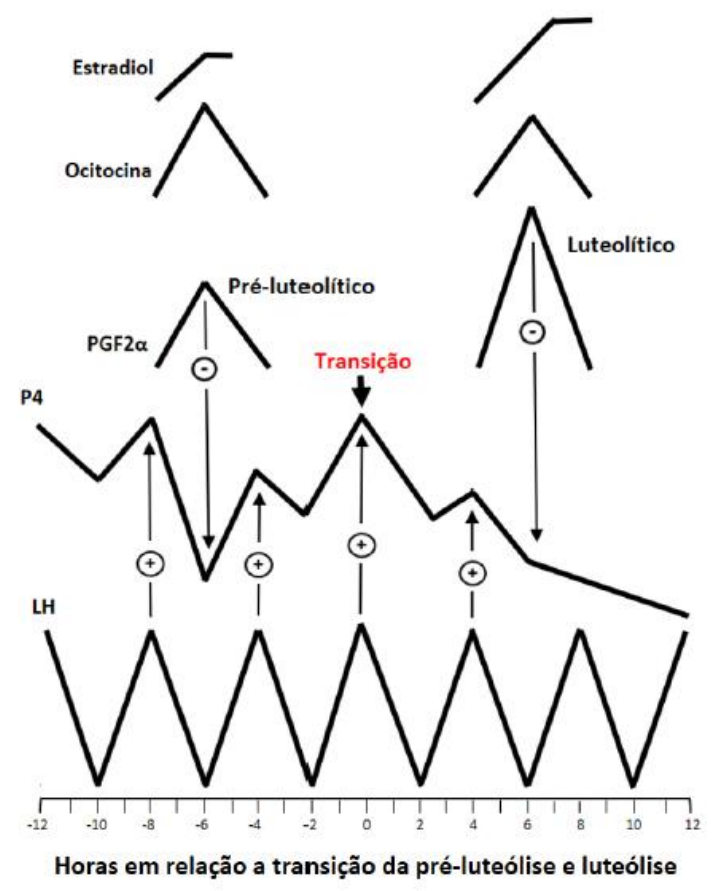

Figura 1 - Modelo esquemático das interações das concentrações de estradiol e dos pulsos de ocitocina, PGF2 $\alpha$ e LH com a secreção de P4 durante o período pré-luteolítico e luteolítico em bovinos (Adaptado de Ginther et al. 2011b).

\section{FUNÇÃO DA PROLACTINA}

Além dos hormônios supracitados, outros hormônios como a prolactina (PRL) podem exercer funções específicas no mecanismo luteolítico. Em ratos, após o fim da secreção de P4, a PRL induz a apoptose das células luteais para se completar a regressão estrutural (Rothchild, 1981). Ao contrário, tem sido reportado que a infusão in vitro de PRL em ovários bovinos aumenta a secreção de P4 (Bartosik et al, 1967), já em roedores e carnívoros, a PRL é sugerida como o mais importante constituinte no complexo luteotrófico (Murphy et al, 1985).

Locais de ligação para PRL foram reportados (Poindexter et al, 1979) no CL bovino, sugerindo que a PRL possua uma função durante a fase luteal do ciclo estral. A expressão de PRL e de RNAm para seus receptores varia durante a fase luteal (Shibaya et al, 2006) e pode estar relacionada com a regulação da secreção de P4 (Thompson et al, 2011). Adicionalmente, segundo indicado por resultados in vitro (Erdman et al, 2007), a PRL pode atuar como um fator vasoativo, mediando o desenvolvimento $\mathrm{e}$ regressão luteal.

A ocorrência de pulsos proeminentes de PRL foi reportada durante e após a luteólise funcional em novilhas (Ginther; Beg, 2011) e éguas (Ginther et al, 2011d). Em bovinos, esta secreção pulsátil de PRL é mais proeminente e rítmica durante as 12 horas antes do fim da luteólise e durante as primeiras 12 horas da pós-luteólise (Ginther and Beg, 2011). A duração de um pulso de PRL é por volta de 4 horas (Ginther; Beg, 2011). Os pulsos de PRL após o início da luteólise são temporariamente associados com o aumento das concentrações de PGFM em novilhas (Ginther; Beg, 2011) e éguas (Ginther et al, 2011d). Centralizado ao pico de PGFM, a concentração de PRL é maior no pico e após uma hora deste, indicando aparentemente um efeito positivo da PGF2 $\alpha$ na secreção de PRL em bovinos (Ginther; Beg, 2011). Entretanto, ainda não é conhecido se um hormônio estimula o outro ou se outros fatores estão envolvidos nesta relação temporal. 


\section{CONSIDERAÇÕES FINAIS}

A regressão espontânea do corpo lúteo em bovinos envolve uma complexa inter-relação hormonal e sinalização em tecidos neuroendócrinos para que ocorra uma cascata luteolítica coordenada, resultando na perda da função luteal em secretar progesterona e na redução morfológica deste tecido. Tal controle envolve a fundamental participação do útero, hipófise e ovário na secreção de hormônios esteroides (estradiol), peptídeos (ocitocina e prolactina) e eicosanoides (prostaglandina F2 $\alpha$ ). Desta forma, verifica-se pelos diversos estudos supracitados que o evento de luteólise funcional nos bovinos compreende um período ao redor de 24 horas e que a luteólise estrutural pode ser verificada subsequentemente ao início da redução da progesterona circulante através de avaliações ultrassonográficas. A compreensão de tal controle neuroendócrino na manutenção ou perda da funcionalidade luteal é fundamental para se aprofundar o conhecimento deste evento que controla o ciclo estral bovino, e para se aplicar diversas biotécnicas reprodutivas objetivando melhoria na eficiência reprodutiva de rebanhos para produção de leite ou carne.

\section{AGRADECIMENTOS}

Agradecemos o apoio da Fundação de Amparo à Pesquisa do Estado de São Paulo (FAPESP) pela concessão de bolsa e auxílio ao primeiro autor (Processos: 2015/10606-9, 2016/23964-3) e bolsa de mestrado para GDM (processo: 2017/13472-9).

\section{REFERÊNCIAS}

ACOSTA, T. J.; YOSHIZAWA, N.; OHTANI, M.; MIYAMOTO, A. Local changes in blood flow within the early and midcycle corpus luteum after PGF $2 \alpha$ injection in the cow. Biology of Reproduction, v.66, p.651-8, 2002.

ANDERSON, L.L.; BLAND, K. P.; MELAMPY, R. M. Comparative aspects of uterine-luteal relationships. Recent Progress in Hormone Research, v.25, p.57104, 1969.

ARAUJO, R. R.; GINTHER, O. J.; FERREIRA, J. C.; PALHAO, M. M.; BEG, M. A.; WILTBANK, M. C. Role of follicular estradiol-17 $\beta$ in timing of luteolysis in heifers. Biology of Reproduction, v.81, p.426-37, 2009.

AROSH, J. A.; BANU, S. K.; CHAPDELAINE, P.; MADORE, E.; SIROIS, J.; FORTIER, M. A. Prostaglandin biosynthesis, transport, and signaling in corpus luteum: a basis for autoregulation of luteal function. Endocrinology, v.145, p.2551-60, 2004.

BARCIKOWSKI, B.; PICHOVA, D.; SKARDA, J.; JERLICKA, I. The oestrous cycle in cattle after transplantation of the ovary. Proc. Symp. RIA in
Anim. Physiol. Slovak Acad. Sci., Kosice; Czech Acad. Sci., Prague p.89-100, 1976.

BARTOSIK, D.; ROMANOFF, E. B.; WATSON, D. J.; SCRICCO, E. Luteotropic effects of prolactin in the bovine ovary. Endocrinology, v.81, p.186-94, 1967.

BEAVIS, E. L. G.; BROWN, J. B.; SMITH, M. A. Ovarian function after hysterectomy with conservation of the ovaries in premenopausal women. Journal of Obstetrics and Gynaecology, v.76, p.969-78, 1969.

BEG, M. A.; GINTHER, O. J. Follicle selection in cattle and horses: role of intrafollicular factors. Reproduction, v.132, p.365-77, 2006.

BEHRMAN, H. R.; ENDO, T.; ATEN, R.; MUSICKI, B. Corpus luteum function and regression. Reproductive Medicine Review, v.2, p.153-60, 1993.

BENYO, D. F.; PATE, J. L. Tumor necrosis factoralpha alters bovine luteal cell synthetic capacity and viability. Endocrinology, v.130, p.854-960, 1992.

BERTAN, C. M.; BINELLI, M.; MADUREIRA, E. H.; TRALDI, A. S. Mecanismos endócrinos e moleculares envolvidos na formação do corpo lúteo e na luteólise. Brazilian Journal of Veterinary Research and Animal Science, v.43, p.824-840, 2006.

BIRKEDAL-HANSEN, H. Proteolytic remodeling of the extracellular matrix. Current Opinion in Cell Biology, v.7, p.728-35, 1995.

CHEN, D.; FONG, H. W.; DAVIS, J. S. Induction of c-fos and c-jun messenger ribonucleic acid expression by prostaglandin $\mathrm{F} 2 \alpha$ is mediated by a protein kinase $\mathrm{C}$-dependent extracellular signal regulated kinase mitogen-activated protein kinase pathway in bovine luteal cells. Endocrinology, v.142, p.887-95, 2001.

CUPP, A. S.; STUMPF, T. T.; KOJIMA, F. N.; WERTH, L. A.; WOLFE, M. W.; ROBERSON, R. J.; KITTOK, R. J.; KINDER, J. E. Secretion of gonadotropins change during the luteal phase of the bovine estrous cycle without corresponding changes in progesterone or $17 \beta$-estradiol. Animal Reproduction Science, v.37, p.109-19, 1995.

DAVIS, A. J.; FLEET, I. R.; HANSFORD, P. A.; HARRISON, F. A.; WALKER, F. M. M. Pulmonary metabolism of prostaglandin F2 $\alpha$ in the conscious nonpregnant cow. Journal of Physiology, v.358, p.107$118,1985$.

DAVIS, J. S.; WEAKLAND, L. L.; WEILAND, D. A.; FARESE, R. V.; WEST, L. A. Prostaglandin F(2 $\alpha)$ stimulates phosphatidylinositol 4,5-bisphosphate hydrolysis and mobilizes intracellular $\mathrm{Ca}^{2}+$ in bovine luteal cells. Proceedings of the National Academy of Sciences of the United States of America, v.84, p.3728-32, 1987. 
DAVIS, J. S.; RUEDA, B. R. The corpus luteum: an ovarian structure with maternal instincts and suicidal tendencies. Frontiers in Bioscience, v.7, p.1949-78, 2002.

DONALDSON, L. E.; HANSEL, W. Prolongation of life span of the bovine corpus luteum by single injections of bovine luteinizing hormone. Journal of Dairy Science, v.48, p.903-41965, 1965.

DISKIN, M. G.; MURPHY, J. J.; SREENAN, J. M. Embryo survival in dairy cows managed under pastoral conditions. Animal Reproduction Science, v.96, p.297-311, 2006.

FAIRCLOUGH, R. J.; MOORE, L. G.; MCGOWAN, L. T.; PETERSON, A. J.; SMITH, J. F.; TERVIT, H. R.; WATKINS, W. B. Temporal relationship between plasma concentrations of 13,14-dihydro-15- ketoprostaglandin F and neurophysin I/II around luteolysis in sheep. Prostaglandins, v.20, p.199-208, 1980.

FLINT, A. P. F.; SHELDRICK, E. L. Ovarian secretion of oxytocin is stimulated by Prostaglandins. Nature, v.297, p.587-8, 1982.

GINTHER, O. J; WOODY, C. O.; JANAKIRAMAN, K.; CASIDA, L. E. Effect of intrauterine plastic coil on the oestrous cycle of the heifer. Journal of Reproduction and Fertility, v.12, p.193-8, 1966.

GINTHER, O. J. Local versus systemic uteroovarian relationships in farm animals. Acta Veterinaria Scandinavica, v.77, p.103-15, 1981.

GINTHER, O. J. Ultrasonic Imaging and Animal Reproduction: Book 4, Color-Doppler Ultrasonography. Cross Palins, WI. Equiservices Publishing; 2007.

GINTHER, O. J; SILVA, L. A.; ARAUJO, R. R.; BEG, M. A. Temporal associations among pulses of 13,14-dihydro-15-keto-PGF2alpha, luteal blood flow, and luteolysis in cattle. Biology of Reproduction, v.76, p.506-13, 2007.

GINTHER, O. J; ARAUJO, R. R.; PALHAO, M. P.; RODRIGUES, B. L.; BEG, M. A. Necessity of sequential pulses of prostaglandin $\mathrm{F} 2 \alpha$ for complete physiologic luteolysis in cattle. Biology of Reproduction, v.80, p.641-8, 2009a.

GINTHER, O. J; SIDDIQUI, M. A.; BEG, M. A. Physiologic and nonphysiologic effects of exogenous prostaglandin F2 $\alpha$ on reproductive hormones in mares. Theriogenology, v.72, p.417-24, 2009b.

GINTHER, O. J; SHRESTHA, H. K.; FUENZALIDA, M. J.; SHAHIDUZZAMAN, A. K.; HANNAN, M. A.; BEG, M. A. Intrapulse temporality between pulses of a metabolite of prostaglandin F2 $\alpha$ and circulating concentrations of progesterone before, during, and after spontaneous luteolysis in heifers. Theriogenology, v.74, p.1179-86, 2010a.
GINTHER, O. J; SHRESTHA, H. K.; FUENZALIDA, M. J.; SHAHIDUZZAMAN, A. K.; BEG, M. A. Characteristics of pulses of 13,14-dihydro-15ketoprostaglandin F2 $\alpha$ before, during, and after spontaneous luteolysis and temporal intrapulse relationships with progesterone concentrations in cattle. Biology of Reproduction, v.82, p.1049 -56, 2010 b.

GINTHER, O. J; SHRESTHA, H. K.; BEG, M. A. Circulating concentrations within a pulse of a metabolite of prostaglandin F2 $\alpha$ during preluteolysis and early luteolysis in heifers. Animal Reproduction Science, v.122, p.253-8, 2010c.

GINTHER, O. J; SHRESTHA, H. K.; FUENZALIDA, M. J.; BEG, M. A. Stimulation of pulses of 13,14dihydro-15-keto-PGF2 $\alpha$ (PGFM) with estradiol-17 $\beta$ and changes in circulating progesterone concentrations within a PGFM pulse in heifers. Theriogenology, v.74, p.384-92, 2010d.

GINTHER, O. J; FUENZALIDA, M. J.; SHRESTHA, H. K.; BEG, M. A. Concomitance of luteinizing hormone and progesterone oscillations during the transition from preluteolysis to luteolysis in cattle. Domestic Animal Endocrinology, v.75, p.164-71, 2010e.

GINTHER, O. J; RODRIGUEZ, M. B.; BEG, M. A. Concentrations of circulating hormones during the interval between pulses of a PGF $2 \alpha$ metabolite in mares and heifers. Animal Reproduction Science, v.128, p.22-28, 2011 b.

GINTHER, O. J; HANNAN, M. A.; BEG, M. A. Luteolysis and associated interrelationships among circulating PGF2, progesterone, LH, and estradiol in mares. Domestic Animal Endocrinology, v.41, p.174184, 2011a.

GINTHER, O. J; FUENZALIDA, M. J.; SHRESTHA, H. K.; BEG, M. A. The transition between preluteolysis and luteolysis in cattle. Theriogenology, v.75, p.164-71, $2011 \mathrm{~b}$.

GINTHER, O. J; PINAFFI, F. L. V.; SILVA, L. A.; BEG, M. A. Temporal relationship of a pulse of prolactin (PRL) to a pulse of metabolite of PGF2 $\alpha$ in mares. Theriogenology, v.77, p.99-107, 2011d.

GINTHER, O. J; BEG, M. A. Pulses of prolactin before, during, and after luteolysis and synchrony with pulses of metabolite of prostaglandin F2 $\alpha$ in heifers. Animal Reproduction Science, v.128, p.29-36, 2011.

HIXON, J. E.; HANSEL, W. Evidence for preferential transfer of prostaglandin F2 $\alpha$ to the ovarian artery following intrauterine administration in cattle. Biology of Reproduction, v.11, p.543-52, 1974.

HOOPER, S. B.; WATKINS, W. B.; THORBURN, G. D. Oxytocin, oxytocin-associated neurophysin, and prostaglandin F2 $\alpha$ concentrations in the utero-ovarian vein of pregnant and nonpregnant sheep. Endocrinology, v.119, p.2590-7, 1986. 
IMAM, S.; SHRESTHA, H. K.; BEG, M. A.; GINTHER, O. J. Intrapulse changes in progesterone and LH concentrations and luteal blood flow during an estradiol-induced pulse of a metabolite of prostaglandin F2 $\alpha$ in heifers. Animal Reproduction Science, v.121, p.34-8, 2010.

IVELL, R.; FUCHS, A. R.; BATHGATE, R.; TILLMANN, G.; KIMURA, T. Regulation of oxytocin receptor in bovine reproductive tissues and the role of steroids. Reproduction of Domestic Animals, v.35, p.134-41, 2000.

KAWANO, T.; OKAMURA, H.; TAJIMA, C.; FUKUMA, K.; KATABUCHI, H. Effect of RU 486 on luteal function in the early pregnant rat. Journal of Reproduction and Fertility, v.83, p.279-85, 1988.

KINDHAL, H.; LINDELL, J. O.; EDQVIST, L. E. Release of prostaglandin F2 $\alpha$ during oestrus cycle. Acta Veterinaria Scandinavica, v.77, p.143-58, 1981.

KOTWICA, J.; SKARZYNSKI, D.; JAROSZEWISKI, J.; WILLIAMS, G.L.; BOGACHI, M. Uterine secretion of PGF2 $\alpha$, stimulated by different doses of oxytocin and released spontaneously during luteolysis in cattle. Reproduction Nutrition Development, v.38, p.217-226, 1998.

LAMSA, J. C.; KOT, S. J.; ELDERING, J. A.; NAY, M. G.; MCCRACKEN, J. A. Prostaglandin F2 $\alpha-$ stimulated release of ovarian oxytocin in the sheep in vivo: threshold and dose dependency. Biology of Reproduction, v.40, p.1215-23, 1989.

LAUDERDALE, J. W.; FOKOLOWSKY, J. H. Efficacy of Lutalyse sterile solution. In: Proceedings from the Lutalyse Symposium; August 6-8; Augusta, Michigan p.26-7, 1979.

LEFRANCE, M.; GOFF, A. K. Effects of progesterone and estradiol 17- $\beta$ on oxytocin-induced release of prostaglandin F2 $\alpha$. Journal of Reproduction and Fertility, v.82, p.429-36,1988.

LEI, Z. M.; CHEGINI, N.; RAO, C.V. Quantitative cell composition of human and bovine corpora lutea from various reproductive states. Biology of Reproduction, v.44, p.1148-1156, 1991.

LOEB, L. Effects of hysterectomy on system of sex organs and on periodicity of sexual cycle in guinea pigs. American Journal of Physiology, v.83, p.20224, 1927.

LOEB, L. The effect of extirpation of the uterus on the life and function of the corpus luteum in the guinea pig. Proceedings of the Society for Experimental Biology and Medicine, v.20, p.441-3, 1923.

LOUIS, T. M.; HAFS, H. D.; MORROW, D. A. Intrauterine administration of prostaglandin $\mathrm{F} 2 \alpha$ in cows: progesterone, estrogen, LH, estrus and ovulation. Journal of Animal Science, v.38, p.347-53, 1974.
MANN, G. E.; LAMMING, G. E. Use of repeated biopsies to monitor endometrial oxytocin receptors in the cow. Veterinary Record, v.135, p.403-5, 1994.

MANN, G. E.; LAMMING, G. E. Timing of prostaglandin PGF2 $\alpha$ release episodes and oxytocin receptor development during luteolysis in the cow. Animal Reproduction Science, v.93, p.328-36, 2006.

MANN, G. E.; PAYNE, J. H.; LAMMING, G. E. Hormonal regulation of oxytocin-induced prostaglandin PGF2 $\alpha$ secretion by the bovine and ovine uterus in vivo. Domestical Animal Endocrinology, v.2, p.127-41, 2001.

MCCRACKEN, J. A.; SCHRAMM, W.; OKULICZ, W. C. Hormone receptor control of pulsatile secretion of PGF2 $\alpha$ from the ovine uterus during luteolysis and its abrogation in early pregnancy. Animal Reproduction Science, v.7, p.31-55, 1984.

MCCRACKEN, J. A.; CUSTER, E. E.; LAMSA, J. C.; ROBINSON, A. G. The central oxytocin pulse generator: a pacemaker for luteolysis. Advances in Experimental Medicine and Biology, v.395, p.133154,1995 .

MCCRACKEN, J. A.; CUSTER, E. E.; LAMSA, J. C. Luteolysis: a neuroendocrine-mediated event. Physiological reviews, v.79, p.263-323, 1999.

MEYER, H. D. D.; MITTERMEIER, T. H.; SCHAMS, D. Dynamics of oxytocin, estrogen and progestin receptors in the bovine endometrium during the estrous cycle. Acta Endocrinologica v.118, p.96-104, 1988.

MILVAE, R. A. Inter-relationships between endothelin and prostaglandin F $2 \alpha$ in corpus Luteum function. Rev. Reproduction, v.5, p.1-5, 2000.

MIYAMOTO, A.; VON LUTZOW, H.; SCHAMS, D. Acute actions of prostaglandin F2 $\alpha$, E2, and I2 in microdialyzed bovine corpus luteum in vitro. Biological Reproduction, v.49, p.423-430, 1993.

MIYAMOTO, A.; SHIRASUNA, K.; SASAHARA, K. Local regulation of corpus luteum development and regression in the cow: Impact of angiogenic and vasoactive factors. Domestical Animal Endocrinology, v.37, p.159-169, 2009.

MOORE, L. G.; CHOY, V. J.; ELLIOT, R.L.; WATKINS, W. B. Evidence for the pulsatile release of PGF2 $\alpha$ inducing the release of ovarian oxytocin during luteolysis in the ewe. Journal of Reproduction and Fertility, v.76, p.159-66,1986.

MURPHY, B. D.; RAJKUMAR. K. Prolactin as a luteotrophin. Canadian Journal of Physiology and Pharmacology, v.63, p.257-64, 1985.

NEILL JD, JOHANSSON ED, KNOBIL E. Failure of hysterectomy to influence the normal pattern of cyclic progesterone secretion in the rhesus monkey. Endocrinology, v.84, p.464-5, 1969. 
NISWENDER, G. D.; JUENGEL, J. L.; SILVA, P. J.; ROLLYSON, M. K.; MCINTUSH, E. W. Mechanisms controlling the function and life span of the corpus luteum. Physiological reviews, v.80, p.1-29, 2000.

NISWENDER, G. D.; REIMERS, T. J.; DIEKMAN, M.A.; NETT, T. M. Blood flow: a mediator of ovarian function. Biology of Reproduction, v.14, p.64-81, 1976.

O'SHEA, J. D.; RODGERS, R.J.; D'OCCHIO, M. J. Cellular composition of the cyclic corpus luteum of the cow. Journal of Reproduction and Fertility, v.85, p.483-7, 1989.

POINDEXTER, A. N.; BUTTRAM JR, V. C.; BESCH, P. K.; SMITH, R. G. Prolactin receptors in the ovary. Fertility and Sterility, v.31:273-7, 1979.

PROCKNOR, M.; DACHIR, S.; OWENS, R. E.; LITTLE, D. E.; HARMS, P. G. Temporal relationship of the pulsatile fluctuation of luteinizing hormone and progesterone in cattle: a time series cross-correlation analysis. Journal of Animal Science, v.62, p.191-8, 1986.

QUINTAL-FRANCO, J. A.; KOJIMA, F. N.; MELVIN, E. J.; LINDSEY, B. R.; ZANELLA, E.; FIKE, K. E.; WEHRMAN, M. E.; CLOPTON, D. T.; KINDER, J. E. Corpus luteum development and function in cattle with episodic release of luteinizing hormone pulses inhibited in the follicular and early luteal phases of the estrous cycle. Biology of Reproduction, v.61, p.921-6, 1999.

ROTHCHILD, I. The regulation of the mammalian corpus luteum. Recent Progress in Hormone Research, v.37; p.183-298, 1981.

SAlleS, M. G. F.; ARAúJO, A. A. Corpo lúteo cíclico e gestacional: revisão. Revista Brasileira de Reprodução Animal, v. 34, p.185-94. 2010.

SCHRAMM, W.; BOVAIRD, L.; GLEW, M. E.; SCHRAMM, G.; MCCRACKEN, J. A. Corpus luteum regression induced by ultra-low pulses of prostaglandin F2 $\alpha$. Prostaglandins, v.26, p.347-64, 1983.

SHIBAYA, M.; MURAKAMI, S.; TATSUKAWA, Y.; SKARZYNSKI, D. J.; ACOSTA, J. T.; OKUDA, K. Bovine corpus luteum in an extrapituitary site of prolactin production. Molecular Reproduction and Development, v.73, p.512-9, 2006.

SHIRASUNA, K.; SHIMIZU, T.; HAYASHI, K-G.; NAGAI, K.; MATSUI, M.; MIYAMOTO, A. Positive association, in local release, of luteal oxytocin with endothelin 1 and prostaglandin f2alpha during spontaneous luteolysis in the cow: a possible intermediatory role for luteolytic cascade within the corpus luteum. Biology of Reproduction, v.76, p.96570,2007
SHIRASUNA, K. Nitric oxide and luteal blood flow in the luteolytic cascade in the cow. Journal of Reproduction and Development, v.56, p.9-14, 2010.

SHRESTHA, H. K.; BEG, M. A.; SIDDIQU, M. A.; GINTHER, O. J. Dynamic progesterone responses to simulation of a natural pulse of a metabolite of prostaglandin F $2 \alpha$ in heifers. Animal Reproduction Science, v.118, p.118-23, 2010a.

SHRESTHA, H. K.; BEG, M. A.; IMAM, S.; GINTHER, O. J. Luteal blood flow and concentrations of circulating progesterone and other hormones associated with a simulated pulse of 13,14-dihydro-15keto-prostaglandin F2 $\alpha$ in heifers. Reproduction, v.139, p.673-83, 2010 b.

SHRESTHA, H. K.; PUGLIESI, G.; BEG, M. A.; GINTHER, O. J. Role of LH in changes in concentrations of progesterone and lutealblood flow during the hours of a simulated pulse of 13,14-dihydro15-keto-prostaglandin F2 $\alpha$ (PGFM) in heifers. Biology of Reproduction, v.85, p.482-9, 2011.

SILVIA, W. J.; LEWIS, G. S.; MCCRACKEN, J. A.; THATCHER, W. W.; WILSON, L. Hormonal regulation of uterine secretion of prostaglandin F2 $\alpha$ during luteolysis in ruminants. Biology of Reproduction, v.45, p.655-63, 1991.

Silvia WJ, Raw RE. Regulation of pulsatile secretion of prostaglandin F2 $\alpha$ from the ovine uterus by ovarian steroids. J Reprod Fert 1993;98:341-7.

SILVIA, W. J.; TAYLOR, M. E. Relationship between uterine secretion of prostaglandin $\mathrm{F} 2 \alpha$ induced by oxytocin and endogenous concentrations of estradiol and progesterone at three stages of the bovine estrous cycle. Journal of Animal Science, v.67, p.2347-53, 1989.

SKARZYNSKI, D. J.; FERREIRA-DIAS, G.; OKUDA, K. Regulation of luteal function and corpus luteum regression in cows: hormonal control, immune mechanisms and intercellular communication. Reproduction of Domestic Animals, v.43, p.57-65, 2008.

SMITH, G. W.; JUENGEL, J. L.; MCINTUSH, E. W.; YOUNGQUIST, R. S.; GARVERICK, H. A.; SMITH, M. F. Ontogenies of messenger RNA encoding tissue inhibitor of metalloproteinases 1 and 2 within bovine periovulatory follicles and luteal tissue. Domestical Animal Endocrinology, v.13, p.151-60, 1996.

STOCCO, C.; TElleRIA, C.; GIBORI, G. The molecular control of corpus luteum formation, function, and regression. Endocrine Reviews, v.28, p.117-149, 2007.

STRAUSS, J. F.; STAMBAUGH, R. L. Induction of $20 \alpha$-hydroxysteroid dehydrogenase in rat corpora lutea of pregnancy by prostaglandin F2 $\alpha$. Prostaglandins, v.5, p.73-85, 1974. 
TAI, C. J.; KANG, S. K.; CHOI, K. C.; TZENG, C. R.; LEUNG, P. C. Role of mitogen-activated protein kinase in prostaglandin $\mathrm{F}(2 \alpha)$ action in human granulosa-luteal cells. Journal of Clinical Endocrinology Metabolism, v.86, p.375-80, 2001.

THATCHER, W. W.; GUZELOGLU, A.; MATTOS, R.; BINELlI, M.; HANSEN, T. R.; PRU, J. K. Uterine-conceptus interactions and reproductive failure in cattle. Theriogenology, v. 56, p.1435-50, 2001.

THATCHER, W. W.; TERQUI, M.; THIMONIER, J.; MAULEON, P. Effect of estradiol-17 $\beta$ on peripheral plasma concentration of 15-keto-13,14-dihydro PGF2 $\alpha$ and luteolysis in cyclic cattle. Prostaglandins, v.31, p.745-756, 1986.

THOMPSON, I. M.; OZAWA, M.; BUBOLZ, J. W.; YANG, Q.; DAHL, G. E. Bovine luteal prolactin receptor expression: Potential involvement in regulation of progesterone during the estrous cycle and pregnancy. Journal of Animal Science, v.89, p.133846, 2011 .

WALTERS, D. L.; SCHAMS, D.; SCHALLENBERGER, E. Pulsatile secretion of gonadotropins, ovarian steroids and ovarian oxytocin during the luteal phase of the oestrous cycle of the cow. Journal of Reproduction and Fertility, v.71, p.479-91, 1984
WARD, K. E.; LONGWELL, L. C.; KREIDER, J. L.; GODKE, R. A. Effect of unilateral hysterectomy on cycling beef heifers. Journal of Animal Science, v.43, p.309, 1976.

WATHES, D. C.; HAMON, M. Localization of oestradiol, $\mathrm{P} 4$ and oxytocin receptors in the uterus during the oestrus cycle and early pregnancy of the ewe. Journal of Endocrinology, v.138, p.479-91, 1993.

WEBB, R.; MITCHELL, M. D.; FALCONER, J.; ROBINSON, J. S. Temporal relationships between peripheral plasma concentrations of oxytocin, progesterone and 13,14-dihydro-15-keto-prostaglandin F2 $\alpha$ during the oestrus cycle and early pregnancy in the ewe. Prostaglandins, v.22; p.443-53; 1981.

WEBB, R.; WOAD, K. J.; ARMSTRONG, D. J. Corpus luteum function: local control mechanisms. Domestic Animal Endocrinology, v.23, p.277-285, 2002.

WEEMS, C. W.; WEEMS, Y. S.; RANDEL, R. D. Prostaglandins and reproduction in female farm animals. Vet J, v.171, p.206-28, 2006.

ZINGG, H. H.; GRAZZINI, E.; BRETON, C. ; LARCHER, A. ; ROZEN, F. ; RUSSO, C. ; GUILLON, G.; MOUILLAC, B. Review. Genomic and non-genomic mechanisms of oxytocin receptor regulation. Adv Exp Med Biol, v.449, p.287-95, 1998. 